\title{
Time Encoding Machines With Multiplicative Coupling, Feedforward, and Feedback
}

\author{
Aurel A. Lazar, Fellow, IEEE
}

\begin{abstract}
We introduce a novel class of time encoding machines (TEMs) that exhibit multiplicative coupling, and, feedforward and feedback. We show that a machine with multiplicative coupling is I/O equivalent with an integrate-and-fire neuron with a variable threshold sequence. The same result holds for a TEM with feedforward while a machine with feedback is $\mathrm{I} / \mathrm{O}$ equivalent with an asynchronous sigma/delta modulator with variable thresholds. For all TEMs, an input band-limited signal can be perfectly recovered from the zero crossings of the modulated signal and the threshold sequence. We present the optimal decoding algorithm and give conditions for perfect signal recovery.
\end{abstract}

Index Terms-Feedback, feedforward, perfect signal recovery, time change, time encoding.

\section{INTRODUCTION}

$\mathbf{T}$ IME encoding of a band-limited function $u=u(t), t \in \mathbb{R}$, is a representation of $u$ as a sequence of strictly increasing times $\left(t_{k}\right), k \in \mathbb{Z}$, where $\mathbb{R}$ and $\mathbb{Z}$ denote the set of real numbers and integers, respectively. Alternatively, the output of the encoder is a signal $z=z(t), t \in \mathbb{R}$, with zeros at times $t_{k}$, $k \in \mathbb{Z}$. A time encoding machine (TEM) is the realization of an asynchronous time encoding mechanism. A time decoding machine (TDM) is the realization of an algorithm for signal recovery with arbitrary accuracy.

The interest in time encoding in communications is driven by the expected paradigm shift in the design and implementation of future analog-to-digital converters (ADCs) from information representation in the amplitude domain to information representation in the time domain. Due to the ever decreasing size of integrated circuits and the attendant low voltage, amplitude-domain high-precision quantizers are more and more difficult to implement. TEMs leverage the phenomenal device speeds of which a temporal code can take advantage of [7]. The interest in temporal encoding in neuroscience is closely linked with the natural representation of sensory stimuli (signals) as a sequence of action potentials (spikes). Spikes are discrete time events that carry information about stimuli.

Two classes of invertible TEMs have been investigated in the literature. The first, the asynchronous sigma/delta modulator arising in communications was shown in [3] to be an invertible time encoding mechanism that robustly represents information with respect to parameter variations arising in analog VLSI implementations [2]. The integrate-and-fire neuron arising in neu-

Manuscript received September 1, 2005; revised February 18, 2006. This paper was recommended by Associate Editor J. Suykens.

The author is with the Department of Electrical Engineering, Columbia University, New York, NY 10027 USA (e-mail: aurel@ee.columbia.edu).

Digital Object Identifier 10.1109/TCSII.2006.876462

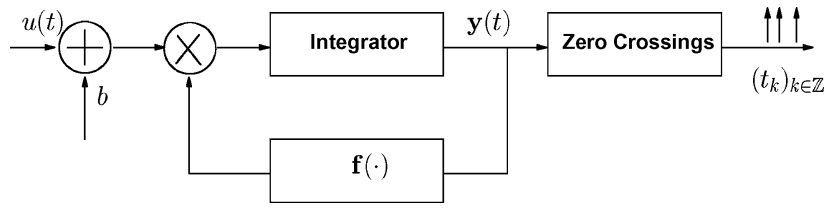

Fig. 1. TEM with multiplicative coupling.

roscience belongs to the second class of TEMs. Both the leaky as well as the integrate-and-fire neuron with a refractory period were shown to be invertible in [4] and [5].

In this paper, we introduce a general class of TEMs that exhibit multiplicative coupling, feedforward, and feedback. The basic TEM with multiplicative coupling consists of a garden-variety oscillator whose output feeds a zero-crossings detector. The detector generates the time sequence of the zeros of the oscillator waveform. The oscillator is in turn modulated by an input band-limited signal.

We show that a TEM with multiplicative coupling is I/O equivalent with an integrate-and-fire neuron with variable threshold. The variable threshold sequence is given by the difference between the consecutive zeros of one of the waveforms generated by the oscillator for unit input. The same result holds for a TEM with feedforward while a TEM with feedback is $\mathrm{I} / \mathrm{O}$ equivalent with an asynchronous sigma/delta modulator with variable thresholds. For all TEMs considered, we demonstrate that the input band-limited signal can be perfectly recovered from the zero crossings of the modulated signal and the threshold sequence. We provide an algorithm for perfect signal recovery.

The TEMs investigated here provide a rich class of circuits for implementing ADCs and generalized frequency modulation schemes for sensor networks. The theoretical methodology presented provides the first rigorous $\mathrm{I} / \mathrm{O}$ equivalence results for nonlinear systems. It also unifies various modulation schemes arising in communications and neuroscience.

\section{TEMs With MultiPlicative COUPLiNG}

The TEMs with multiplicative coupling considered in this study consist of two building blocks (see Fig. 1). The first building block models the operation of a garden-variety oscillator (i.e., an oscillator that generates a stable limit cycle [1]). The second building block generates a set of time events, called trigger times, from the output of the oscillator building block.

In the presence of a constant unit input, the output of the oscillator is described by a set of state space equations

$$
\frac{d \mathbf{x}}{d t}=\mathbf{f}(\mathbf{x})
$$


where $\mathbf{x} \in \mathbb{R}^{n}$ and $\mathbf{f} \in \mathbb{R}^{n}$ are column vectors and $\mathbf{f}: \mathbb{R}^{n} \rightarrow \mathbb{R}^{n}$ is a continuous function. We shall assume that, for an arbitrary initial condition $\mathbf{x}(0)=\mathbf{x}_{0}$, the set of differential equations above has an unique solution; see [1] for details. The zeros of $x_{1}$, the first coordinate of $\mathbf{x}$, denoted by $\left(\delta_{k}\right), k \in \mathbb{Z}$, are also called trigger times. The model mechanism for generating the zeros will be revisited in Section III below.

Let $u=u(t), t \in \mathbb{R}$, be a bounded continuous function on $\mathbb{R}$ with $|u| \leq c ; u+b$ models the input signal to the TEM. With multiplicative coupling, the output of the oscillator building block is given by

$$
d \mathbf{y} / d t=(b+u(t)) \mathbf{f}(\mathbf{y})
$$

where $\mathbf{y} \in \mathbb{R}^{n}$ is a column vector and $b>c$ is a constant. Thus, $b+u(t)>0$, for all $t, t \in \mathbb{R}$. The zero-crossings building block in Fig. 1 detects the zeros of $y_{1}(t)$. These zeros are denoted by $\left(t_{k}\right), k \in \mathbb{Z}$.

Remark 1: The defining building blocks of the TEM with multiplicative coupling have each been employed in a number of modulation schemes in the past. For example, the oscillator building block of the TEM with multiplicative coupling described by (2) also arises in generalized frequency modulation [8]. The zero-crossings building block was previously employed in irregular sampling [6].

Lemma 1: Given the initial condition $\mathbf{y}(0)=\mathbf{x}(0)$, we have

$$
\mathbf{y}=\mathbf{x}\left(b t+\int_{0}^{t} u(s) d s\right)
$$

for all $t, t \in \mathbb{R}_{+}$, where $\mathbf{x}=\mathbf{x}(t), t \in \mathbb{R}_{+}$, is the solution to (1) starting at $\mathbf{x}_{0}$. we obtain

Proof: By differentiating the right-hand side of (3) above,

$$
\begin{aligned}
\frac{d \mathbf{y}}{d t} & =\left.\frac{d \mathbf{x}(v)}{d v}\right|_{v=b t+\int_{0}^{t} u(s) d s} \cdot(b+u(t)) \\
& =(b+u(t)) \cdot \mathbf{f}(\mathbf{y}) .
\end{aligned}
$$

Since $\mathbf{y}(0)=\mathbf{x}(0)$, the assertion follows.

Remark 2: The solution to (2) is derived from the solution to (1) via the time change $t \rightarrow b t+\int_{0}^{t} u(s) d s$. In this light, the condition $b+u(t)>0$ is very natural since it ensures that the changed time remains increasing.

\section{PERFECT RECOVERY}

In what follows, we shall assume that the observable output of the oscillator building block is exactly one of the coordinates of $\mathbf{x}$. Without any loss of generality, we will consider this coordinate to be $x_{1}$. The zeros of $x_{1}(t)$ are denoted by $\left(\delta_{k}\right), k \in \mathbb{Z}$. Therefore

$$
x_{1}\left(\delta_{k}\right)=0
$$

for all $k, k \in \mathbb{Z}$. Recall that the trigger times $\left(t_{k}\right), k \in \mathbb{Z}$, are the zeros of $y_{1}(t)$.

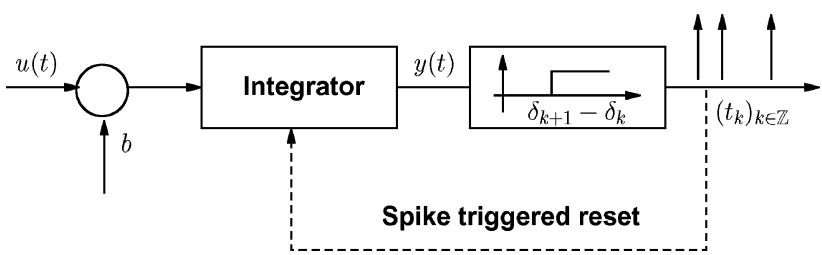

Fig. 2. Integrate-and-fire neuron with variable threshold.

Lemma 2: The set of trigger times $\left(t_{k}\right), k \in \mathbb{Z}$, and the set of zeros $\left(\delta_{k}\right), k \in \mathbb{Z}$, verify the set of recursive equations

$$
\int_{t_{k}}^{t_{k+1}} u(s) d s=\delta_{k+1}-\delta_{k}-b\left(t_{k+1}-t_{k}\right)
$$

for all $k, k \in \mathbb{Z}$.

Proof: Since $\left(\delta_{k}\right), k \in \mathbb{Z}$, are the set of zeros of $x_{1}$, and the zeros of $y_{1}$ are given by $\left(t_{k}\right), k \in \mathbb{Z}$, the equation

$$
x_{1}\left(b t_{k}+\int_{0}^{t_{k}} u(s) d s\right)=0
$$

implies that

$$
b t_{k}+\int_{0}^{t_{k}} u(s) d s=\delta_{k}
$$

and the result follows.

Equation (5) above defines the t-transform [3]; it maps the amplitude information of $(u(t)), t \in \mathbb{R}$, into the time sequence $\left(t_{k}\right), k \in \mathbb{Z}$. Thus, encoding information with a TEM with multiplicative coupling is equivalent with encoding information with an integrate-and-fire neuron [4] with variable threshold $\left(\delta_{k+1}-\delta_{k}\right), k \in \mathbb{Z}$. Both lead to the same trigger time sequence $\left(t_{k}\right)$ for all $k, k \in \mathbb{Z}$. Formally, we have the following proposition.

Proposition 1: Assume that the variable threshold sequence of an integrate-and-fire neuron is identical to the difference between the consecutive zeros of the oscillator waveform generated for unit input. Then, the TEM with multiplicative coupling and the integrate-and-fire neuron are I/O equivalent.

In other words, the TEM depicted in Fig. 1 and the integrateand-fire neuron with variable threshold represented in Fig. 2 are $\mathrm{I} / \mathrm{O}$ equivalent (the integrator reset value is zero).

\section{A. Recovery Algorithms}

Informally, a linear function of the length of the interval between two consecutive trigger times provides, via the t-transform, an estimate of the integral of $u(t)$ on the same interval. For a finite energy signal $u$, this estimate used in conjunction with the band-limited and boundedness assumption on the same, enables a perfect reconstruction of the signal even though the trigger times are irregular. In order to achieve perfect reconstruction, the distance between two consecutive trigger times 
has to be, on average [4], [5], smaller than the distance between the uniformly spaced samples in the classical sampling theorem.

The mathematical methodology for signal recovery employed here is based on finding, under appropriate conditions, the inverse of the $t$-transform. This inverse perfectly recovers from the time sequence $\left(t_{k}\right), k \in \mathbb{Z}$, the amplitude information of the signal $u=u(t), t \in \mathbb{R}$. Our derivation below closely follows [3].

Let $\Xi$ be the space of square integrable functions defined on $\mathbb{R}$ that are band-limited to $[-\Omega, \Omega]$ (clearly $\Xi \subset L^{2}(\mathbb{R})$ ). We shall construct an operator $\mathcal{A}: \Xi \rightarrow \Xi$ and, by starting from a good initial guess followed by successive iterations, obtain successive approximations that converge in the $L^{2}$-norm to the original signal $u$.

Let us assume that $u=u(t), t \in \mathbb{R}$, with $|u(t)| \leq c<b$, is a function in $\Xi$ and let the operator $\mathcal{A}$ be given by

$$
\mathcal{A} u=\sum_{k \in \mathbb{Z}} \int_{t_{k}}^{t_{k+1}} u(s) d s g\left(t-s_{k}\right),
$$

where $g(t)=\sin (\Omega t) / \pi t$ and $s_{k}=\left(t_{k+1}+t_{k}\right) / 2$.

The realization of the operator $\mathcal{A}$ above is highly intuitive. Dirac-delta pulses generated at times $s_{k}$ with weight $\int_{t_{k}}^{t_{k+1}} u(s) d s=\delta_{k+1}-\delta_{k}-b\left(t_{k+1}-t_{k}\right)$ are passed through an ideal low-pass filter with unity gain for $\omega \in[-\Omega, \Omega]$ and zero otherwise. Note that the values of $\left(\int_{t_{k}}^{t_{k+1}} u(s) d s\right), k \in \mathbb{Z}$, are obtained, via the $t$-transform, from the sequence $\left(t_{k}\right), t \in \mathbb{Z}$, and are available at the TDM.

Let $u_{l}=u_{l}(t), t \in \mathbb{R}$, be a sequence of band-limited functions defined by the recursion

$$
u_{l+1}=u_{l}+\mathcal{A}\left(u-u_{l}\right)
$$

for all $l, l \in \mathbb{N}$, with the initial condition $u_{0}=\mathcal{A} u$. The supremum of the distance between two consecutive zeros in the unitary input case is denoted by $\delta$, i.e., $\delta=\sup _{k \in \mathbb{Z}}\left(\delta_{k+1}-\delta_{k}\right)$. Let us define the vectors $\mathbf{g}=\left[g\left(t-s_{k}\right)\right], \mathbf{q}=\left[\delta_{k+1}-\delta_{k}-\right.$ $\left.b\left(t_{k+1}-t_{k}\right)\right]$ and the matrix $\mathbf{G}=\left[G_{l k}\right]=\left[\int_{t_{l}}^{t_{l+1}} g\left(s-s_{k}\right) d s\right]$; I denotes the identity matrix and $T$ the (matrix) transpose. In addition, we assume that $\cup_{k \in \mathbb{Z}}\left[\delta_{k}, \delta_{k+1}\right)=\mathbb{R}$.

Theorem 1 (t-transform Inverse): Let $\left(\delta_{k}\right)$ and $\left(t_{k}\right), k \in \mathbb{Z}$, be the trigger times of the TEM with multiplicative coupling with unit input and $b+u(t), t \in \mathbb{R}_{+}$, respectively. If $\delta<$ $(b-c)(\pi / \Omega)$, then the signal $u$ can be perfectly recovered from the trigger times $\left(t_{k}\right)$ and $\left(\delta_{k}\right), k \in \mathbb{Z}$, as

$$
\begin{aligned}
\lim _{l \rightarrow \infty} u_{l}(t) & =u(t) \\
\left\|u-u_{l}\right\| & \leq r^{l+1}\|u\|
\end{aligned}
$$

where $r=(\delta / b-c)(\Omega / \pi)$. Furthermore, $u_{l}(t)=\mathbf{g}^{T} \mathbf{P}_{l} \mathbf{q}$, where $\mathbf{P}_{l}$ is given by $\mathbf{P}_{l}=\sum_{k=0}^{l}(\mathbf{I}-\mathbf{G})^{k}$. Finally

$$
u(t)=\sum_{k \in \mathbb{Z}} c_{k} g\left(t-s_{k}\right)
$$

with $\mathbf{c}=\mathbf{G}^{+} \mathbf{q}$, where $\mathbf{G}^{+}$denotes the pseudo-inverse of $\mathbf{G}$.

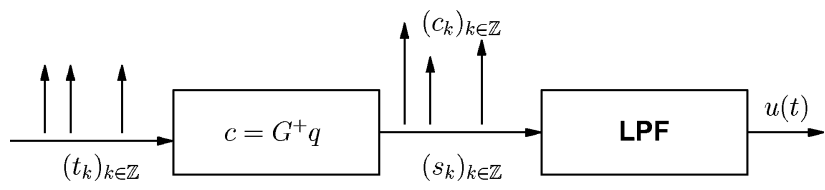

Fig. 3. Block diagram representation of the recovery algorithm.

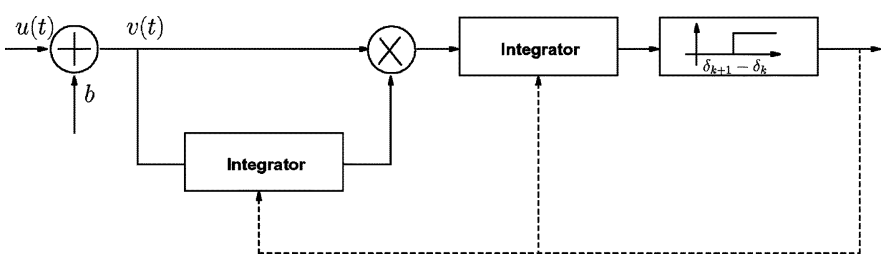

Fig. 4. TEM with multiplicative coupling and feedforward.

Proof: The proof is based on observing that operator $\mathcal{A}$ and the matrix $G$ verify the equality $(I-\mathcal{A})^{k} \mathbf{g}^{T}=\mathbf{g}^{T}(\mathbf{I}-\mathbf{G})^{k}$. See [3] for further details. The signal recovery algorithm given by (9) has a very simple representation as shown in Fig. 3.

\section{TEMs WITH FEEDFORWARD AND FEEDBACK}

Here, we consider two extensions of the class of TEMs with multiplicative coupling. First, we introduce a number of feedforward schemes. Second, we present a simple feedback mechanism that is representative for other feedback schemes. The $\mathrm{t}$-transform for both the feedforward and feedback schemes can easily be derived using the chain rule for derivatives.

\section{A. TEMs With Feedforward}

A TEM with a feedforward circuit accepts a processed version of the band-limited signal $u=u(t), t \in \mathbb{R}$, as its input.

Example 1: Assume that the input to a TEM with multiplicative coupling on the time interval $\left[t_{k}, t_{k+1}\right]$ is given by $v(t) \int_{t_{k}}^{t} v(s) d s$, where $v(t)=b+u(t)>0$ for all $t, t \in \mathbb{R}$. Fig. 4 shows the block diagram representation of the feedforward circuit followed by the TEM with multiplicative coupling. For simplicity, only the block diagram of the I/O equivalent integrate-and-fire neuron is shown in the figure. The TEM with multiplicative coupling and feedforward is described for all $k$, $k \in \mathbb{Z}$ by

$$
\int_{t_{k}}^{t_{k+1}} v(s) \int_{t_{k}}^{s} v(\sigma) d \sigma d s=\delta_{k+1}-\delta_{k}
$$

or

$$
\int_{t_{k}}^{t_{k+1}} u(s) d s=\left[2\left(\delta_{k+1}-\delta_{k}\right)\right]^{1 / 2}-b\left(t_{k+1}-b_{k}\right) .
$$

Thus, the TEM with feedforward shown in Fig. 4 is I/O equivalent with an integrate-and-fire neuron with variable threshold sequence $\left[2\left(\delta_{k+1}-\delta_{k}\right)\right]^{1 / 2}, k \in \mathbb{Z}$. 


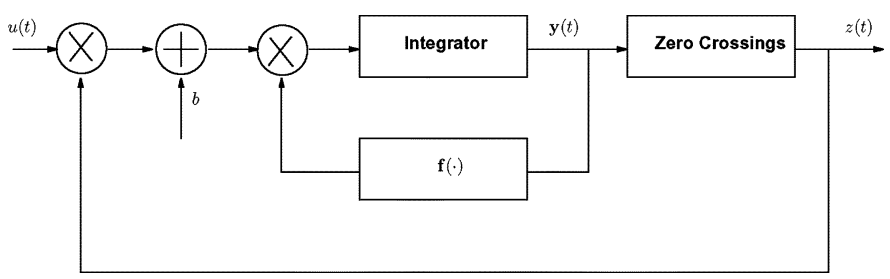

Fig. 5. TEM with multiplicative coupling and feedback.

More generally, assume that the $t$-transform describing the operation of the TEM with a feedforward circuit is described on the interval $\left[t_{k}, t_{k+1}\right]$ by

$$
h\left(\int_{t_{k}}^{t_{k+1}} v(s) d s\right)=\delta_{k+1}-\delta_{k}
$$

where $h$ is an arbitrary function on $\mathbb{R}$. If (10) has a solution of the form

$$
\int_{t_{k}}^{t_{k+1}} v(s) d s=h^{-1}\left(\delta_{k+1}-\delta_{k}\right)
$$

then the original band-limited signal $u$ can be again perfectly recovered from

$$
\int_{t_{k}}^{t_{k+1}} u(s) d s=h^{-1}\left(\delta_{k+1}-\delta_{k}\right)-b\left(t_{k+1}-t_{k}\right)
$$

provided that $r=(\delta /(b-c))(\Omega / \pi)<1$, with $\delta=$ $\sup _{k \in \mathbb{Z}} h^{-1}\left(\delta_{k+1}-\delta_{k}\right)$. Thus, we have the following proposition.

Proposition 2: A TEM with multiplicative coupling and feedforward is I/O equivalent with an integrate-and-fire neuron with variable threshold. The variable threshold sequence of the neuron can be explicitly derived from the zeros of the oscillator's waveform for unit input.

\section{B. TEMs With Feedback}

The TEMs with feedback introduced in this section derive their feedback from the output of the zero crossings building block. For the simple examples considered here, the t-transform of the TEMs with feedback can be reduced to the t-transform describing the asynchronous sigma/delta modulator [3].

An example of a TEM with (multiplicative) feedback is shown in Fig. 5. The feedback is easily implemented by composing the input $z(t) u(t)+b$, where $z(t)=\operatorname{sgn}\left(y_{1}(t)\right)$ for all $t, t \in \mathbb{R}$. Assuming by convention that $z(t)=1$ for all $t$, $t \in\left[t_{0}, t_{1}\right]$, the t-transform of the TEM in Fig. 5 is given by

$$
\int_{t_{k}}^{t_{k+1}} u(s) d s=(-1)^{k}\left[\delta_{k+1}-\delta_{k}-b\left(t_{k+1}-t_{k}\right)\right]
$$
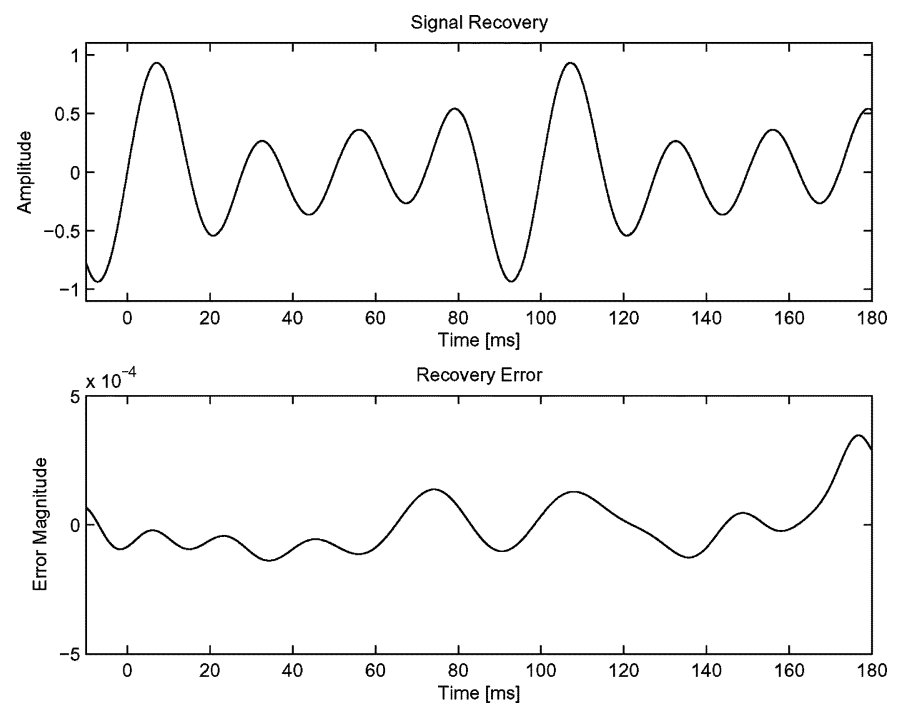

Fig. 6. Error recovery of a band-limited signal.

for all $k, k \in \mathbb{Z}$. We recognize in the above equation the $\mathrm{t}$-transform of an asynchronous sigma-delta modulator [3] with variable thresholds. For the recovery of the band-limited signal $u$, the algorithm presented in Section III-A can be used.

Proposition 3: A TEM with multiplicative coupling and feedback is I/O equivalent with an asynchronous sigma/delta modulator with variable thresholds.

\section{BUILDING TEMS}

TEMs with multiplicative coupling can be built using a wide variety of oscillators, including the harmonic oscillator, the Hodgkin-Huxley neuron, and the Van der Pol oscillator. The only requirement for these oscillators is that the Nyquist-type rate condition $r<1$ remains valid.

Example 2: By using a Van der Pol relaxation oscillator in the first building block of Fig. 1, we obtain the generalized frequency modulation scheme of [8]. The oscillator is described by

$$
\begin{aligned}
& d x_{1} / d t=x_{2} \\
& d x_{2} / d t=\beta\left(1-x_{1}^{2}\right) x_{2}-\alpha^{2} x_{1} .
\end{aligned}
$$

For $\alpha=500$ and $\beta=0.5$, this nonlinear system of equations has a periodic attractor. For the input $u=0.2 \sin (2 \pi 20 t)+$ $0.3 \sin (2 \pi 30 t)+0.5 \sin (2 \pi 40 t)$, a recovery error below $5 \cdot 10^{-4}$ can easily be achieved (see Fig. 6). The recovered signal and the original are virtually indistinguishable. The system was initialized at $(1,0)$, and the results evaluated on the time interval $[25$, 187.5] ms.

\section{CONCLUSION}

We considered the representation of band-limited signals in the time domain using a novel class of TEMs with multiplicative coupling, feedback, and feedforward. The representation was shown to be invertible. From the time-domain sequence, the signal can be perfectly recovered. The methodology presented here can also be employed to extract information contained in 
all of the observable waveforms generated by the TEM oscillator using more general parallel and serial processing circuits.

\section{REFERENCES}

[1] H. K. Khalil, Nonlinear Systems, 3rd ed. Upper Saddle River, NJ: Prentice-Hall, 2002.

[2] P. R. Kinget, A. A. Lazar, and L. T. Toth, "On the robustness of the VLSI implementation of a time encoding machine," in Proc. ISCAS, Kobe, Japan, May 23-26, 2005, pp. 4221-4224.
[3] A. A. Lazar and L. T. Toth, "Perfect recovery and sensitivity analysis of time encoded bandlimited signals," IEEE Trans. Circuits Syst. I: Reg. Papers, vol. 51, no. 10, pp. 2060-2073, Oct. 2004.

[4] A. A. Lazar, "Time encoding with an integrate-and-fire neuron with a refractory period," Neurocomput., vol. 58-60, pp. 53-58, 2004.

[5] A. A. Lazar, "Multichannel time encoding with integrate-and-fire neurons," Neurocomput., vol. 65-66, pp. 401-407, 2005.

[6] F. A. Marvasti, A unified approach to zero-crossings and nonuniform sampling Nonuniform, Oak Park, IL, 1987.

[7] E. Roza, "Analog-to-digital conversion via duty-cycle modulation," IEEE Trans. Circuits Syst. II, Analog Digit. Signal Process., vol. 44, no. 11, pp. 907-914, Nov. 1997.

[8] W. P. Torres, A. V. Oppenheim, and R. R. Rosales, "Generalized frequency modulation," IEEE Trans. Circuits Syst. I, Fundam. Theory Appl., vol. 48, no. 12, pp. 1405-1412, Dec. 2001. 